\title{
Aspects of magnetotunnelling drawn from ab-initio-type calculations
}

\author{
P. Weinberger $\dagger^{*}$, V. Drchal $\dagger \ddagger$ J. KudrnovskÝ $\dagger \dagger$, I. TureK $\dagger$, \\ H. Herper $\dagger$, L. Szunyogh $\dagger \|$ and C. Sommers $\|$ \\ $\dagger$ Center for Computational Materials Science, Technical University of Vienna, \\ Getreidemarkt 9/134, A-1060 Vienna, Austria \\ $\ddagger$ Institute of Physics, Academy of Sciences of the Czech Republic, Na Slovance 2, \\ CZ-182 21 Prague 8, Czech Republic \\ $\S$ Institute of Physics of Materials, Academy of Sciences of the Czech Republic, \\ Žižkova 22, CZ-616 62 Brno, Czech Republic \\ || Department of Theoretical Physics, Institute of Physics, Budapest University of \\ Technology and Economics, Budafoki út 8., H-1521 Budapest, Hungary \\ - Laboratoire de Physique des Solides, Université de Paris-Sud, 91405 Orsay \\ Cedex, France
}

[Received 5 November 2001 and accepted 26 November 2001]

\begin{abstract}
Tunnelling between two semi-infinite ferromagnetic electrodes separated by vacuum is studied theoretically on an ab-initio level by using the fully relativistic spin-polarized screened Korringa-Kohn-Rostoker and the spin-polarized nonrelativistic tight-binding linear muffin-tin orbital method. The KuboGreenwood equation is employed to calculate the non-local conductivity and layer-resolved sheet resistances; the transmission matrix formulation in turn is used to evaluate the conductance. We show that the dependence of sheet resistance on the imaginary part of the Fermi energy allows us to distinguish between the tunnelling and conductive regimes of electron transport. Our numerical studies of the system bcc $\mathrm{Fe}(100) /$ vacuum/bcc $\mathrm{Fe}(100)$ show quite dramatic variations in the electrostatic potential in the vacuum region and a gradual development of the tunnelling barrier with increasing thickness of the vacuum barrier. Varying the Madelung potential in the interior of the vacuum barrier allowed us to simulate spacers made of semiconducting or insulating material and to discuss the conditions for the presence of a magnetoresistance in such multilayers. As far as the thickness of the vacuum barrier is concerned the results obtained with the transmission matrix approach for fcc $\mathrm{Co}(001)$ /vacuum/ fcc $\operatorname{Co}(001)$ show a similar tendency to those obtained within the KuboGreenwood equation for bcc $\mathrm{Fe}(100) /$ vacuum/bcc $\mathrm{Fe}(100)$; asymptotically, that is with increasing thickness of the vacuum barrier, the magnetoresistance tends to zero.
\end{abstract}

\section{$\S 1$. INTRODUCTION}

At present, enormous scientific and commercial interest is devoted to spin-dependent tunnelling between ferromagnetic electrodes separated by a semiconducting or insulating barrier such as in $\mathrm{Fe} / \mathrm{MgO} / \mathrm{Fe}$ or $\mathrm{Fe} / \mathrm{ZnSe} / \mathrm{Fe}$. These systems can be produced with relative ease and bear great promise for technological applications.

*Email: pw@cms.tuwien.ac.at

Philosophical Magazine B ISSN 1364-2812 print/ISSN 1463-6417 online (C) 2002 Taylor \& Francis Ltd 
In order to calculate their electronic and transport properties, however, reliable structural information about the interfaces between the metal and the insulator is required. So far such information based on direct experimental data is not available. Only very recently has a set of criteria for tunnelling in magnetic-insulatormagnetic structures been discussed (Akerman et al. 2001) which concluded that 'tunnelling is rare'. Also very recently an interesting theoretical discussion of the use of the Kubo formula and its relation to the Landauer approach was presented (Nikolić 2001) (see also Nikolić and Allen $(1999,2000)$ ). At the moment, at least in terms of ab-initio-type approaches, one has to resort to various structural models or, as we do in this study, assume a simplified structural model in which the insulating spacer is replaced by a vacuum.

In the following we first summarize briefly the theoretical concepts applied for layered systems, namely the Kubo-Greenwood approach in terms of the screened Korringa-Kohn-Rostoker (KKR) method, and the transmission matrix formulation of the conductance via the tight-binding (TB) linear muffin-tin orbital (LMTO) method. The section containing the numerical results is meant to illustrate main aspects of the tunnelling through a vacuum barrier. In particular the comparison between the two different kinds of approach applied is to confirm the main conclusion of this paper concerning the functional behaviour of the magnetoresistance regarding the thickness of the vacuum barrier.

\section{§ 2. The Kubo-Greenwood EQUATION For LAYERED SySTEMS}

Within the Kubo (1957) - Greenwood (1958) approach the diagonal elements of the conductivity tensor are given (Butler 1985) by

$$
\sigma_{\mu \mu}=\frac{1}{4}\left[\sigma_{\mu \mu}\left(\varepsilon^{+}, \varepsilon^{+}\right)+\sigma_{\mu \mu}\left(\varepsilon^{-}, \varepsilon^{-}\right)-\sigma_{\mu \mu}\left(\varepsilon^{+}, \varepsilon^{-}\right)-\sigma_{\mu \mu}\left(\varepsilon^{-}, \varepsilon^{+}\right)\right],
$$

with $\mu \in(x, y, z)$ and $\varepsilon^{ \pm}=\varepsilon_{\mathrm{F}} \pm \mathrm{i} \delta(\delta \rightarrow 0)$. For a layered system with $n$ atomic layers matched properly via surface Green function techniques to left and right substrates (leads), equation (1) can be reformulated (Weinberger et al. 1996) as

$$
\begin{aligned}
& \sigma_{\mu \mu}(\mathbf{C} ; n)=\frac{1}{n} \sum_{p, q=1}^{n} \sigma_{\mu \mu}^{p q}(\mathbf{C} ; n), \\
& \sigma_{\mu \mu}^{p q}(\mathbf{C} ; n)=\frac{1}{4} \sum_{i, j=1}^{2}(-1)^{i+j} \sigma_{\mu \mu}^{p q}\left(\mathbf{C} ; \varepsilon_{i}, \varepsilon_{j} ; n\right),
\end{aligned}
$$

where $\mathrm{C}$ denotes a particular magnetic configuration, $\varepsilon_{1} \equiv \varepsilon^{+}, \varepsilon_{2} \equiv \varepsilon^{-}$, and in the absence of disorder (for a generalization to disordered systems, see Weinberger et al. (1996)) the $\sigma_{\mu \mu}^{p q}\left(\mathbf{C} ; \varepsilon_{\alpha}, \varepsilon_{\beta} ; n\right)$ are defined by

$$
\sigma_{\mu \mu}^{p q}\left(\mathbf{C} ; \varepsilon_{i}, \varepsilon_{j} ; n\right)=\frac{A}{\Omega_{\mathrm{SBZ}}} \int \operatorname{Tr}\left[J_{\mu}^{p}\left(\mathbf{C} ; \varepsilon_{j}, \varepsilon_{i}\right) \tau^{p q}\left(\mathbf{C} ; \mathbf{k}, \varepsilon_{i}\right) J_{\mu}^{q}\left(\mathbf{C} ; \varepsilon_{i}, \varepsilon_{j}\right) \tau^{q p}\left(\mathbf{C} ; \mathbf{k}, \varepsilon_{j}\right)\right] \mathrm{d}^{2} k .
$$

In $A$, all occurring fundamental constants are collected, the $\tau^{p q}\left(\mathbf{C} ; \mathbf{k}, \varepsilon_{i}\right)$ are the $\mathbf{k}$ resolved layer off-diagonal scattering path operators (Weinberger and Szunyogh 2000 ) respectively, $\Omega_{\mathrm{SBZ}}$ is the unit area of the surface Brillouin zone (SBZ), and in the relativistic case the current matrices are given (Weinberger et al. 1996) by 


$$
\begin{gathered}
J_{\mu}^{p}\left(\mathrm{C} ; \varepsilon_{i}, \varepsilon_{j}\right)=\left\{J_{\mu, \Lambda \Lambda^{\prime}}^{p}\left(\mathrm{C} ; \varepsilon_{i}, \varepsilon_{j}\right)\right\}, \\
J_{\mu, \Lambda \Lambda^{\prime}}^{p}\left(\mathrm{C} ; \varepsilon_{i}, \varepsilon_{j}\right)=e c \int_{W S} Z_{\Lambda}^{p}\left(\mathrm{C} ; \mathbf{r}_{p 0}, \varepsilon_{i}\right)^{\dagger} \alpha_{\mu} Z_{\Lambda^{\prime}}^{p}\left(\mathrm{C} ; \mathbf{r}_{p 0}, \varepsilon_{j}\right) \mathrm{d}^{3} r_{p 0},
\end{gathered}
$$

where $\Lambda$ are appropriate angular momentum quantum numbers, $e$ is the charge of the electron, $c$ the speed of light, the $\alpha_{\mu}$ are the Dirac matrices, and the $Z_{\Lambda}^{p}\left(C ; \mathbf{r}_{p 0}, \varepsilon_{i}\right)$ are regular scattering solutions (Weinberger 1990).

It should be noted that, with respect to the discussion given by Nikolić (2001), equation (1) contains all cross-products and therefore does not correspond to an approximate form of the Kubo equation. The only assumption made is that in the case of perpendicular transport the restriction $|\mathbf{E}|=V / L$ for the electric field $\mathbf{E}$ (Nikolić 2001) is assumed to be valid, where $V$ is the bias voltage ( $V \rightarrow 0$ in the linear transport regime) and $L$ is the (finite) length of the sample, that is $\mathbf{E}$ is a homogeneous electric field (Kubo et al. 1965, Rammer 1998). Formulated in terms of atomic layers this in turn implies that for $\mu=z$ the electric current $j_{\mu}^{p}(\mathbf{C} ; n)$ in layer $p$ is related to electric field $\mathbf{E}^{q}$ in layer $q$ by the following equation:

$$
j_{\mu}^{p}(\mathbf{C} ; n)=\sum_{q=1}^{n} \sigma_{\mu \mu}^{p q}(\mathbf{C} ; n) \mathbf{E}^{q}(n)=|\mathbf{E}(n)| \sum_{q=1}^{n} \sigma_{\mu \mu}^{p q}(\mathbf{C} ; n) .
$$

\subsection{Perpendicular electric transport via the Kubo-Greenwood equation}

By mapping the conductivity tensor (Levy 1994) $\sigma\left(\mathbf{C} ; z, z^{\prime}\right), z$ and $z^{\prime}$ being continuous variables along the surface normal, on to the corresponding conductivity tensor elements $\sigma_{p q}(\mathbf{C} ; n) \equiv \sigma_{z z}^{p q}(\mathbf{C} ; n)$ for a layered system, such that the algebraic structure is conserved, that is

$$
\int \rho\left(\mathbf{C} ; z, z^{\prime \prime}\right) \sigma\left(\mathbf{C} ; z^{\prime \prime}, z^{\prime}\right)=\delta\left(z-z^{\prime}\right), \quad \sum_{t=1}^{n} \rho_{p t}(\mathbf{C} ; n) \sigma_{t q}(\mathbf{C} ; n)=\delta_{p q},
$$

the sheet resistance

$$
r(\mathrm{C} ; n)=\sum_{p, q=1}^{n} \rho_{p q}(\mathrm{C} ; n)
$$

then serves as a measure for the mapping (Weinberger et al. 2001). In fact, equation (8) can be derived immediately from equation (7) with $|\mathbf{E}(n)|$ serving as a measure:

$$
\sum_{q=1}^{n}\left[\sigma_{p q}(\mathbf{C} ; n)\right]^{-1} j_{q}(\mathbf{C} ; n) \equiv \sum_{q=1}^{n} \rho_{p q}(\mathbf{C} ; n) j_{q}(\mathbf{C} ; n)=|\mathbf{E}(n)| .
$$

Suppose that $\delta$ is the imaginary part of the complex Fermi energy, then

$$
r(\mathbf{C} ; n)=\lim _{\delta \rightarrow 0}[r(\mathbf{C} ; n ; \delta)]=\lim _{\delta \rightarrow 0}\left(\sum_{p, q=1}^{n} \rho_{p q}(\mathbf{C} ; n ; \delta)\right),
$$

where

$$
\sum_{t=1}^{n} \rho_{p t}(\mathbf{C} ; n ; \delta) \sigma_{t q}(\mathbf{C} ; n ; \delta)=\delta_{p q}
$$


Furthermore, layer-resolved sheet resistances $r_{p}(\mathrm{C} ; n ; \delta)$ can be defined as

$$
r_{p}(\mathrm{C} ; n ; \delta)=\sum_{q=1}^{n} \rho_{p q}(\mathrm{C} ; n ; \delta)
$$

such that

$$
r(\mathrm{C} ; n ; \delta)=\sum_{p=1}^{n} r_{p}(\mathrm{C} ; n ; \delta) .
$$

It was recently shown (Weinberger et al. 2001) that for heterojunctions of the type $\ldots / \mathrm{L}_{n} \mathrm{X}_{s} \mathrm{~L}_{n} / \ldots$, where $\mathrm{L}_{n}$ denotes $n$ layers of the electrode material $\mathrm{L}$ and $\mathrm{X}_{s} s$ layers of a suitable spacer $\mathrm{X}$, the corresponding sheet resistance $r(\mathrm{C} ; 2 n+s ; \delta)$ for a given value of $\delta$ and $n \geqslant n_{0}$ varies linearly with respect to $n$ :

$$
\begin{gathered}
r(\mathbf{C} ; 2(n+m)+s ; \delta)=r(\mathbf{C} ; 2 n+s ; \delta)+2 m k_{1}(\mathbf{C} ; \delta), \\
\lim _{\delta \rightarrow 0}\left[k_{1}(\mathbf{C} ; \delta)\right]=0,
\end{gathered}
$$

which for a given $n$ and $s$ is linear in $\delta$ :

$$
r(\mathrm{C} ; 2 n+s ; \delta)=r(\mathrm{C} ; 2 n+s)+\delta k_{2}(\mathrm{C} ; 2 n+s) .
$$

From these two equations follows immediately that for $n \geqslant n_{0}$

$$
\lim _{\delta \rightarrow 0}\left[r\left(\mathbf{C} ; 2\left(n_{0}+m\right)+s ; \delta\right)\right]=r\left(\mathbf{C} ; 2 n_{0}+s\right) .
$$

The magnetoresistance is then given as

$$
\begin{gathered}
R\left(2 n_{0}+s\right)=\frac{r\left(\mathrm{AP} ; 2 n_{0}+s\right)-r\left(\mathrm{P} ; 2 n_{0}+s\right)}{r\left(\mathrm{AP} ; 2 n_{0}+s\right)}, \\
R\left(2 n_{0}+s ; \delta\right)=\frac{r\left(\mathrm{AP} ; 2 n_{0}+s ; \delta\right)-r\left(\mathrm{P} ; 2 n_{0}+s ; \delta\right)}{r\left(\mathrm{AP} ; 2 n_{0}+s ; \delta\right)},
\end{gathered}
$$

where AP and $\mathrm{P}$ explicitly denote the antiparallel and parallel magnetic configurations respectively. It should be noted that, since $R\left(2 n_{0}+s ; \delta\right) \leqslant R\left(2 n_{0}+s\right)$, the quantity $R\left(2 n_{0}+s ; \delta\right)$ can be used for qualitative discussions of the magnetoresistance.

\section{§3. THE TRANSMISSION MATRIX FORMALISM}

Within a non-relativistic spin-polarized TB LMTO description of the transmission matrix formalism (Kudrnovský et al. 2000) the conductance for a particular magnetic configuration $\mathrm{C}$ of a layered system of $n$ properly embedded atomic layers is given by

$$
\Sigma(\mathrm{C} ; n)=\sum_{\sigma= \pm 1 / 2} \Sigma^{\sigma}(\mathrm{C} ; n), \quad \Sigma^{\sigma}(\mathrm{C} ; n)=\frac{e^{2}}{h} \frac{1}{\Omega_{\mathrm{SBZ}}} \int T^{\sigma}\left(\mathrm{C} ; \mathbf{k}, \varepsilon_{\mathrm{F}} ; n\right) \mathrm{d}^{2} k,
$$

where for $T^{\sigma}\left(\mathbf{C} ; \mathbf{k}, \varepsilon_{\mathrm{F}} ; n\right)$ the following expression can be used (Kudrnovský et al. 2000):

$$
\begin{aligned}
T^{\sigma}\left(\mathrm{C} ; \mathbf{k}, \varepsilon_{\mathrm{F}} ; n\right)= & \frac{1}{2} \operatorname{Tr}\left[B_{1}^{\beta, \sigma}\left(\mathrm{C} ; \mathbf{k}, \varepsilon_{\mathrm{F}}\right) g_{1 n}^{\beta, \sigma}\left(\mathrm{C} ; \mathbf{k}, \varepsilon^{+}\right) B_{n}^{\beta, \sigma}\left(\mathbf{C} ; \mathbf{k}, \varepsilon_{\mathrm{F}}\right) g_{n 1}^{\beta, \sigma}\left(\mathrm{C} ; \mathbf{k}, \varepsilon^{-}\right)\right. \\
& \left.+B_{1}^{\beta, \sigma}\left(\mathrm{C} ; \mathbf{k}, \varepsilon_{\mathrm{F}}\right) g_{1 n}^{\beta, \sigma}\left(\mathbf{C} ; \mathbf{k}, \varepsilon^{-}\right) B_{n}^{\beta, \sigma}\left(\mathbf{C} ; \mathbf{k}, \varepsilon_{\mathrm{F}}\right) g_{n 1}^{\beta, \sigma}\left(\mathbf{C} ; \mathbf{k}, \varepsilon^{+}\right)\right] .
\end{aligned}
$$


The layer-diagonal blocks of the inverse of the auxiliary Green function matrix $g_{p q}^{\beta, \sigma}\left(\mathbf{C} ; \mathbf{k}, \varepsilon^{ \pm}\right)$are defined (Turek et al. 1997) in terms of potential functions $P_{p}^{\beta, \sigma}\left(\mathrm{C} ; \varepsilon^{ \pm}\right)$and structure constants $S_{p q}^{\beta}(\mathbf{k})$ :

$$
\left(g^{\beta, \sigma}\left(\mathbf{C} ; \mathbf{k}, \varepsilon^{ \pm}\right)\right)_{p p}^{-1}= \begin{cases}P_{1}^{\beta, \sigma}\left(\mathrm{C} ; \varepsilon^{ \pm}\right)-S_{00}^{\beta}(\mathbf{k})-\Gamma_{1}^{\beta, \sigma}\left(\mathrm{C} ; \mathbf{k}, \varepsilon^{ \pm}\right), & p=1, \\ P_{p}^{\beta, \sigma}\left(\mathrm{C} ; \varepsilon^{ \pm}\right)-S_{00}^{\beta}(\mathbf{k}), & 1<p<n, \\ P_{N}^{\beta, \sigma}\left(\mathrm{C} ; \varepsilon^{ \pm}\right)-S_{00}^{\beta}(\mathbf{k})-\Gamma_{N}^{\beta, \sigma}\left(\mathrm{C} ; \mathbf{k}, \varepsilon^{ \pm}\right), & p=n,\end{cases}
$$

while its off-diagonal blocks $(1 \leqslant p, q \leqslant n)$ are independent of the magnetic configuration and given by

$$
\left(g^{\beta, \sigma}\left(\mathbf{C} ; \mathbf{k}, \varepsilon^{ \pm}\right)\right)_{p q}^{-1}= \begin{cases}-S_{01}^{\beta}(\mathbf{k}), & q=p+1, \\ -S_{10}^{\beta}(\mathbf{k}), & q=p-1, \\ 0, & \text { otherwise. }\end{cases}
$$

It should be noted that these expressions only apply in the case of an infinite parent lattice (Weinberger 1997). The $B_{p}^{\beta, \sigma}\left(\mathbf{C} ; \mathbf{k}, \varepsilon_{\mathrm{F}}\right)$ refer to the so-called embedding potentials $\Gamma_{p}^{\beta, \sigma}\left(\mathbf{C} ; \mathbf{k}, \varepsilon^{ \pm}\right)$:

$$
B_{p}^{\beta, \sigma}\left(\mathrm{C} ; \mathbf{k}, \varepsilon_{\mathrm{F}}\right)=\mathrm{i}\left[\Gamma_{p}^{\beta, \sigma}\left(\mathrm{C} ; \mathbf{k}, \varepsilon^{+}\right)-\Gamma_{p}^{\beta, \sigma}\left(\mathrm{C} ; \mathbf{k}, \varepsilon^{-}\right)\right],
$$

which arise from the matching (Turek et al. 1997) to the left (L) and right (R) semiinfinite systems (leads)

$$
\Gamma_{p}^{\beta, \sigma}\left(\mathrm{C} ; \mathbf{k}, \varepsilon^{ \pm}\right)= \begin{cases}S_{10}^{\beta}(\mathbf{k}) G_{\mathrm{L}}^{\beta, \sigma}\left(\mathrm{C} ; \mathbf{k}, \varepsilon^{ \pm}\right) S_{01}^{\beta}(\mathbf{k}), & p=1 \\ S_{01}^{\beta}(\mathbf{k}) G_{\mathrm{R}}^{\beta, \sigma}\left(\mathrm{C} ; \mathbf{k}, \varepsilon^{ \pm}\right) S_{10}^{\beta}(\mathbf{k}) ; & p=n, \\ 0, & \text { otherwise }\end{cases}
$$

with corresponding surface Green functions $\mathrm{G}_{\mathrm{L}}^{\beta, \sigma}\left(\mathrm{C} ; \mathbf{k}, \varepsilon^{ \pm}\right)$and $\mathrm{G}_{\mathrm{R}}^{\beta, \sigma}\left(\mathrm{C} ; \mathbf{k}, \varepsilon^{ \pm}\right)$.

Comparing equation (21) with equations (10)-(12) in the paper by Nikolic (2001) it is evident that the embedding potentials formally play the role of the left and right imaginary parts of the self-energy there. Quite clearly the advantage of this kind of formulation is that only the Green functions for the end points ( 1 and $n$ ) need to be calculated, both of which have to be within the leads.

\section{§4. Relation to the Kubo-Greenwood approach for perpendicular ELECTRIC TRANSPORT}

As equations (21) and (22) are based on the Kubo-Greenwood equation (see the appendix of the paper by Kudrnovský et al. (2000)), they ought to show certain similarities to equations (1)-(4). For further similarities see also Weinberger et al. (1997) and Turek et al. (2002). The most obvious differences between these two approaches arise from, firstly, the use of a localized (TB) representation, secondly, imposing spin-current conservation, which of course is restricted to a non-relativistic description, and, thirdly, reducing the double sum over layer indices in equation (2) to a single term between two arbitrarily chosen layers from the left and right lead each, namely between those labelled by 1 and $n$. In order to obtain more insight into the relationship between the two formalisms, we define the following auxiliary quantities for perpendicular electric transport by restricting the summation in equation (3) to $p=1$ and $q=n$ : 


$$
\begin{gathered}
\bar{\sigma}(\mathrm{C} ; n)=\frac{1}{4} \sum_{i, j=1}^{2}(-1)^{i+j} \bar{\sigma}_{z z}^{1 n}\left(\mathrm{C} ; \varepsilon_{i}, \varepsilon_{j} ; n\right) \\
\bar{\sigma}_{z z}^{1 n}\left(\mathrm{C} ; \varepsilon_{i}, \varepsilon_{j} ; n\right)=\frac{A}{\Omega_{\mathrm{SBZ}}} \int \operatorname{Tr}\left[J_{z}^{1}\left(\mathrm{C} ; \varepsilon_{j}, \varepsilon_{i}\right) \tau^{1 n}\left(\mathrm{C} ; \mathbf{k}, \varepsilon_{i}\right) J_{z}^{n}\left(\mathrm{C} ; \varepsilon_{i}, \varepsilon_{j}\right) \tau^{n 1}\left(\mathrm{C} ; \mathbf{k}, \varepsilon_{j}\right)\right] \mathrm{d}^{2} k
\end{gathered}
$$

and consequently

$$
\bar{r}(\mathrm{C} ; n)=\frac{1}{\bar{\sigma}_{z z}(\mathrm{C} ; n)},
$$

with indices 1 and $n$ referring to lead layers.

Clearly enough the two-point conductivity in equation (28) is not independent of the choice of the two end points. Therefore, in keeping one end point fixed in a lead layer and the other variable, one can define the following quantity, $p>1$ :

$$
\begin{aligned}
\bar{r}_{(p)}(\mathrm{C} ; n)= & \left(\frac{1}{4} \sum_{i, j=1}^{2}(-1)^{i+j} \bar{\sigma}_{z z}^{1 p}\left(\mathrm{C} ; \varepsilon_{i}, \varepsilon_{j} ; n\right)\right)^{-1}, \\
& \bar{r}_{(n)}(\mathrm{C} ; n)=\bar{r}(\mathrm{C} ; n),
\end{aligned}
$$

which then can be used to trace the role of different layers in perpendicular transport. A formally similar quantity can be defined in terms of the layer-dependent sheet resistances introduced in equation (13):

$$
r_{(p)}(\mathrm{C} ; n)=\sum_{q=1}^{p} r_{q}(\mathrm{C} ; n),
$$

such that

$$
r_{(n)}(\mathrm{C} ; n)=r(\mathrm{C} ; n)
$$

\section{§5. VERTEX CORRECTIONS AND THE COHERENT POTENTIAL APPROXIMATION}

Although this is not the main topic of the present paper, it is worthwhile to comment on the application of the above two approaches for disordered systems. Returning to equations (4) and (22) it is evident that in the case of disorder in the spacer region or in the case of interdiffusion at interfaces in principle the following configurational average has to be performed:

$$
\left\langle A_{i} G_{i j}^{+} A_{j} G_{j i}^{-}\right\rangle=\left\langle A_{i} G_{i j}^{+}\right\rangle(1-\Omega)\left\langle A_{j} G_{j i}^{-}\right\rangle=A_{i}\left\langle G_{i j}^{+}\right\rangle(1-\Omega) A_{j}\left\langle G_{j i}^{-}\right\rangle,
$$

where the $A_{i}$ refer either to current matrices or to the embedding potentials both of which are translationally invariant within a chosen layer $i$ and $G_{i j}^{+}$simply denotes a scattering path operator (see equation (4)) or an auxilary Green function (equation (22)). Even after omitting vertex corrections $(\Omega=0)$ the problem arises of how to average $\left\langle G_{i j}^{ \pm}\right\rangle$. In the case of the coherent potential approximation this is achieved by considering a two-impurity problem, that is by occupying distinctly two sites in the coherent potential medium and then averaging over the occupations of these two sites; for details see Weinberger et al. (1996) and Turek et al. (1997). This, however, is only possible in the case of equation (3) since there is a double sum over all layers 
which of course consists of disordered but also lead layers. Thus by using equation (4) not only do the scattering path operators have to refer to the coherent potential medium, but also all the $\left\langle G_{i j}^{ \pm}\right\rangle$have to be averaged properly. Equation (22) only applies to pure leads; for pure leads there is no (additional) averaging that can be performed for $G_{i j}^{+}, i=1, j=n$, although the elements of the Green function matrix itself refer to the coherent potential medium. Of course this particular problem does not arise when averaging over superlattices (supercell approach), since per definition superlattices are ordered (translationally invariant) systems. Using superlattices implies, however, an average over all possible ordered structures and structural arrangements, a procedure that requires additional computational efforts.

\section{$\S 6$. Computational details}

\subsection{Screened Korringa-Kohn-Rostoker and Kubo-Greenwood calculations}

In this paper the fully relativistic spin-polarized form of the Kubo-Greenwood equation for layered systems (Weinberger et al. 1996, Blaas et al. 1999, 2001) is applied by using $1830 k_{\|}$points in the irreducible wedge of the surface Brillouin zone (ISBZ) for the occurring SBZ integrals and by choosing $n_{0} \geqslant 11$; see also equation (18). In all cases the effective potentials and effective exchange fields were determined self-consistently by means of the fully relativistic spin-polarized screened KKR method (Weinberger and Szunyogh 2000) within the local-density approximation (Vosko et al. 1980) considering a minimum of $45 k_{\|}$points in the ISBZ and by assuming a bcc parent lattice (Weinberger 1997) with a lattice spacing of bcc Fe $\left(a_{0}=5.27 \mathrm{au}\right)$. All self-consistent calculations refer to a ferromagnetic (parallel) magnetic configuration with the orientation of the magnetization parallel to the surface normal.

\subsection{Tight-binding linear muffin-tin orbital transmission matrix calculations}

The transmission matrix formalism is implemented within the framework of the scalar-relativistic TB LMTO method (Kudrnovský et al. 2000). Keeping in mind the presence of the so-called hot spots (Wunnicke et al. 2001) a very large number (980 700) of $k_{\|}$points in the ISBZ were used in order to perform the $k_{\|}$integration properly. We assumed a model of semi-infinite fcc $\mathrm{Co}(001)$ leads separated by a vacuum slab of various thicknesses represented by atomic layers of empty spheres. The lattice spacing was that of the parent fcc Co lattice. The lead potentials were bulk-like potentials of fcc Co while the vacuum potentials corresponded to a vacuum layer far away from a single $\mathrm{Co}$-vacuum interface. We have thus assumed a 'rectangular' tunnelling barrier. Finally, the potentials for the parallel and the antiparallel alignments of the two Co parts of the system were the same, implying that, for the antiparallel configuration, the majority and minority spins only have to be interchanged.

\section{$\S 7$. ReSULTS}

\subsection{Kubo-Greenwood approach for perpendicular electric transport}

In figure 1 the layer-resolved Madelung potentials in bcc $\mathrm{Fe}(100) / \mathrm{Fe}_{n_{0}} /$ vacuum $_{s} /$ $\mathrm{Fe}_{n_{0}} / \mathrm{Fe}(100)$ are shown for $s \leqslant 6$. As can be seen for $s \leqslant 2$ the spill-out of charge is still sufficient to connect the two Fe semi-infinite systems while for $s \geqslant 3$ in the middle of the vacuum barrier a rather large positive barrier is building up. This 

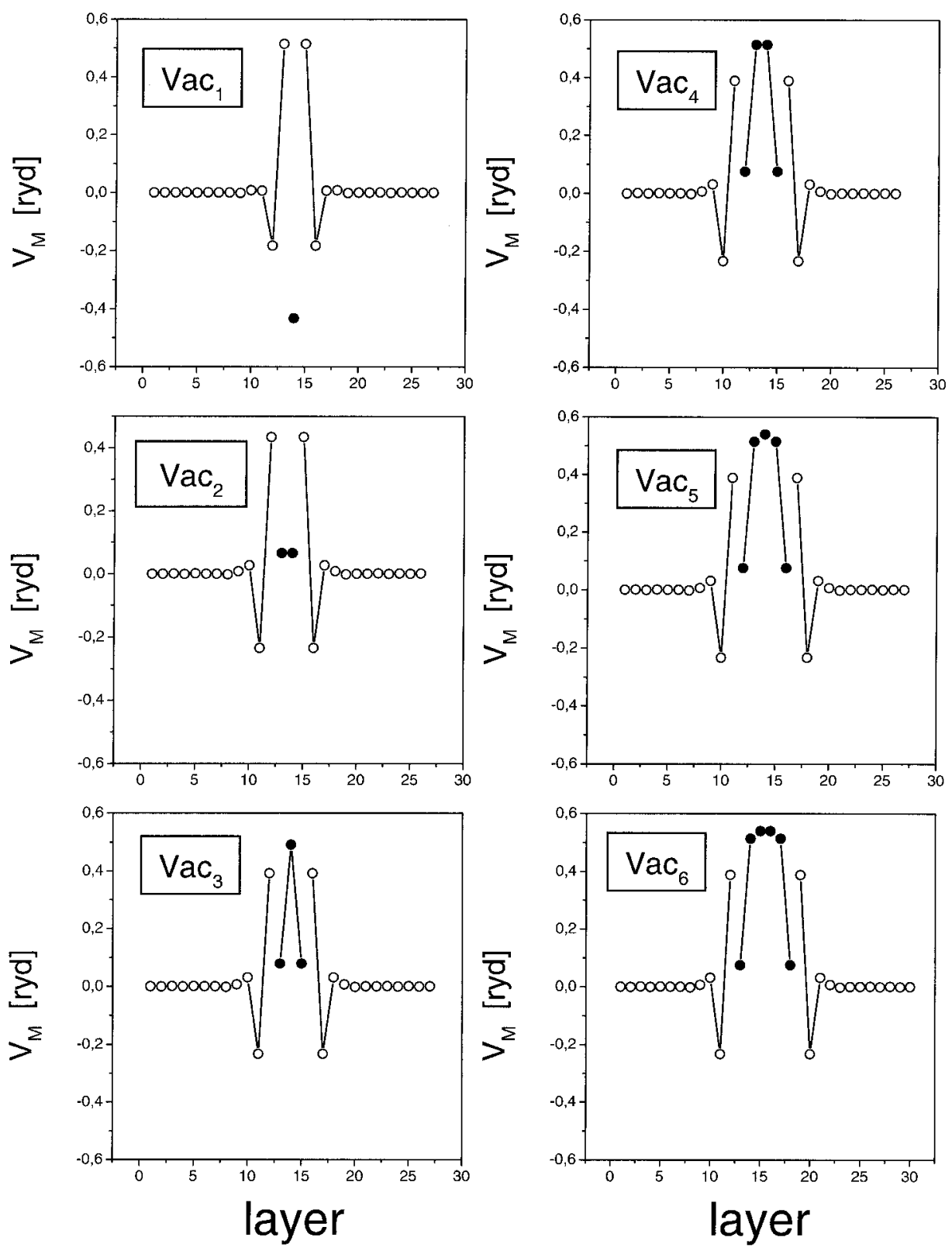

Figure 1. Layer-resolved Madelung potentials in bcc $\mathrm{Fe}(100) / \mathrm{Fe}_{n_{0}} / \operatorname{vacuum}_{s} / \mathrm{Fe}_{n_{0}} / \mathrm{Fe}(100)$, $n_{0} \geqslant 11:(\bigcirc)$, Fe-like contributions, $(\mathbf{O})$, contributions from the vacuum layers. The number $s$ of vacuum layers is indicated explicitly.

positive barrier causes a dramatic increase in the corresponding layer-resolved sheet resistances $r_{p}\left(\mathrm{C} ; 2 n_{0}+s ; \delta\right), \delta=2 \mathrm{mRy}$ (figure 2). For $s \leqslant 2$ the layer-resolved sheet resistances oscillate weakly; the shape of the curves and, in particular, the size of these layer-wise contributions seem to indicate at a first glance metallic-like conductance. For $s \geqslant 3$ a characteristic change in the shapes of the curves happens; in all those layers whose Madelung potentials are sufficiently positive (see figure 1), 

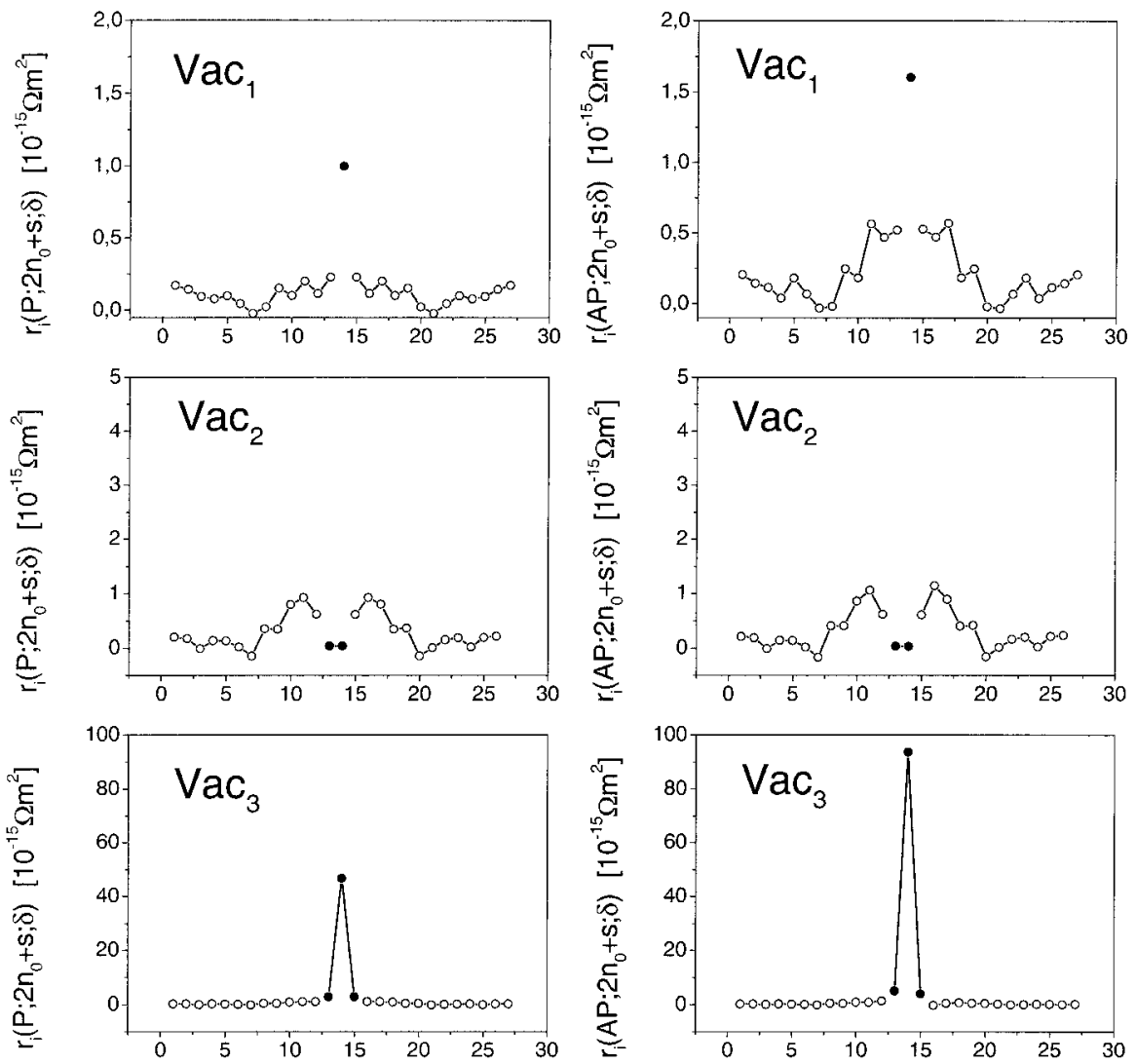

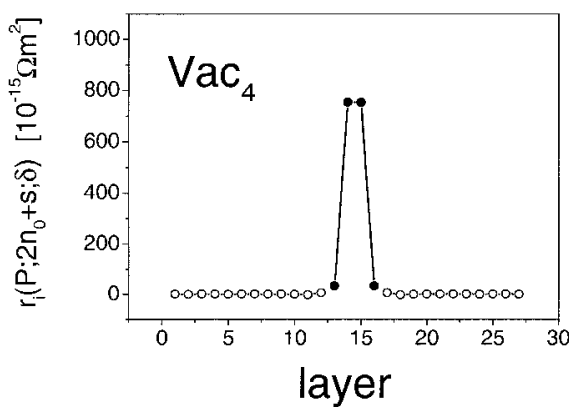

(a)

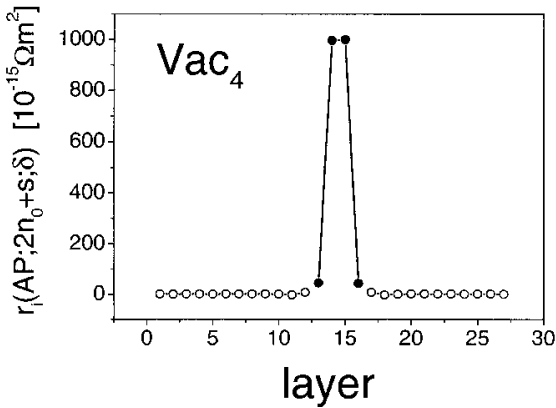

(b)

Figure 2. Layer-resolved sheet resistances $r_{i}\left(\mathrm{C} ; 2 n_{0}+s ; \delta\right), n_{0} \geqslant 11, \delta=2 \mathrm{mRy}$, for $(a)$ the parallel and $(b)$ the antiparallel magnetic configuration: $(\bigcirc)$, Fe-like contributions,

(O), contributions from the vacuum layers. The number $s$ of vacuum layers is indicated explicitly.

the corresponding layer-resolved sheet resistances are very large and grow in size with increasing thickness of the vacuum barrier.

Not only does figure 3 serve as an illustration for the analytical continuation of the sheet resistances $r\left(\mathrm{C} ; 2 n_{0}+s ; \delta\right)$ to the real energy axis but also, depending on the sign of the slope of the sheet resistances with respect to $\delta$ (see also equation (16)), one 
(a)

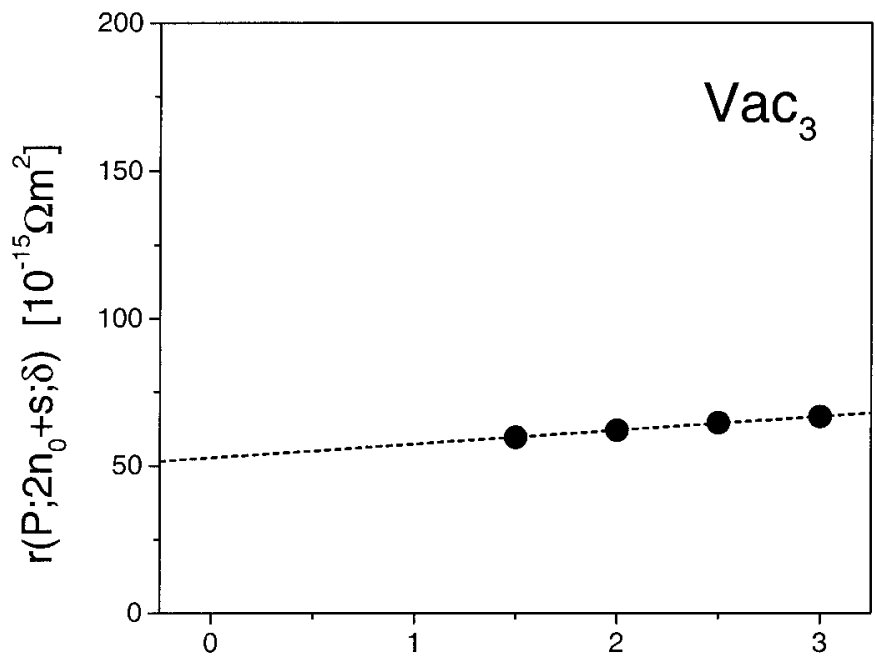

(b)

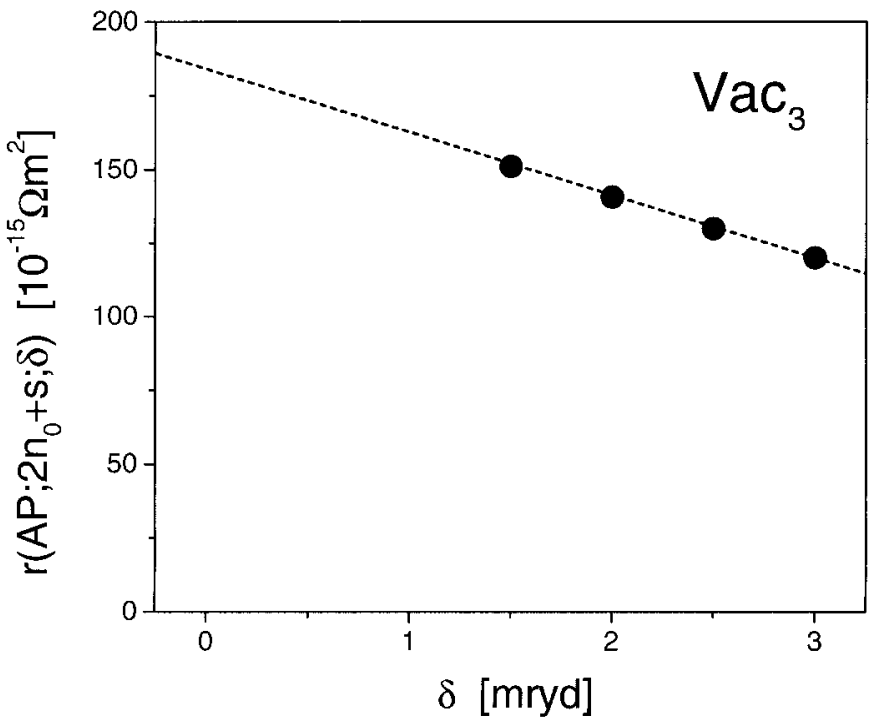

Figure 3. Analytical continuation of the sheet resistances $r\left(\mathrm{C} ; 2 n_{0}+s ; \delta\right), n_{0} \geqslant 11, s=3$, to the real energy axis for $(a)$ the parallel configuration and $(b)$ the antiparallel configuration: $(\bullet)$, calculated values; $(\ldots \ldots)$ corresponding linear fit.

can easily distinguish qualitatively between a metallic and a tunnelling type of electric transport,

$$
k_{2}= \begin{cases}>0, & \text { metallic conductance } \\ <0, & \text { tunnelling conductance }\end{cases}
$$

Interestingly, the above statement can be correlated with the density of states (DOS), $n\left(\varepsilon_{\mathrm{F}}\right)$, at the Fermi level since, for $k_{2}>(<) 0, n\left(\varepsilon_{\mathrm{F}}\right)$ is decreased (increased) with increasing $\delta$. This can be seen in figure 4 in the case when $s=3,6$ and 9. In this figure (note the different scaling factors) the DOS at the Fermi energy is displayed as a 


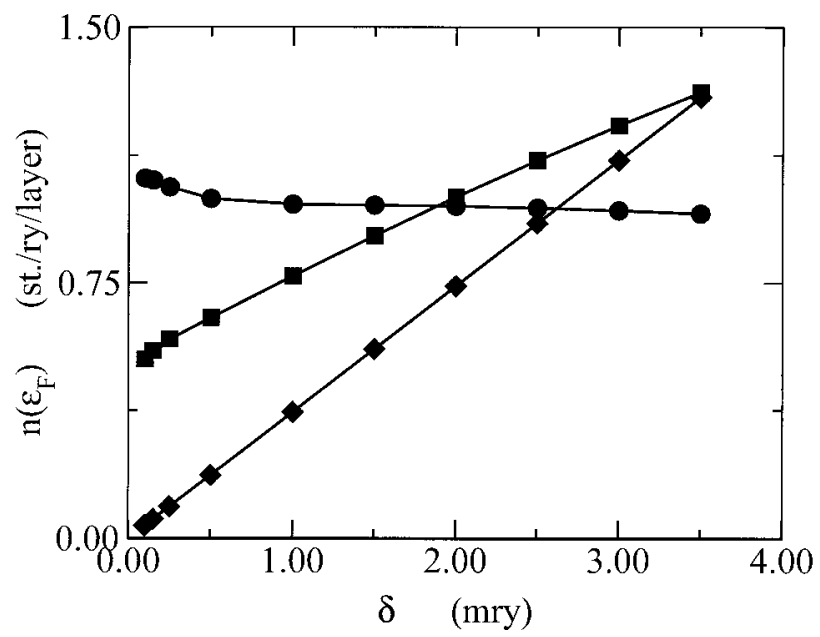

Figure 4. DOS of the centre vacuum layer at the complex Fermi energy $\varepsilon_{\mathrm{F}}+\mathrm{i} \delta$ as a function of $\delta$ in bcc $\mathrm{Fe}(100) / \mathrm{Fe}_{12} / \operatorname{vacuum}_{s} / \mathrm{Fe}_{12} / \mathrm{Fe}(100)$ for $s=3(\mathbf{O}), s=6(\boldsymbol{\square})$ (multiplied by $100)$ and $s=9(\diamond)$ (multiplied by 250$)$.

function of $\delta$ for the centre vacuum layer. From this figure it is also evident that only for $s \geqslant 9$ does the DOS in the centre layer vanish completely at the Fermi energy.

In figure $5(a)$ the logarithm of the sheet resistance in the parallel configuration is displayed for various values of $\delta$ with respect to the number $s$ of vacuum layers; in figure $5(b)$ for a particular value of $\delta$ a comparison between the results obtained by means of either equation (13) or (29) is shown. As can be seen from figure 5 by using the auxiliary quantity defined in equation (29) for $s \geqslant 3, \ln \left[\bar{r}\left(\mathrm{P} ; 2 n_{0}+s ; \delta\right)\right]$ increases linearly with increasing $s$ whereas, in the Kubo-Greenwood type of formulation, $\ln \left[r\left(\mathrm{P} ; 2 n_{0}+s ; \delta\right)\right]$, this is only the case for $3 \leqslant s \leqslant 5$. For $s>5$ the shape of the curve turns into behaviour that is proportional $\ln (s)$. This different 'asymptotic behaviour' results in a totally different behaviour of the magnetoresistance (figure 6), while in the case of the auxiliary quantity for very large values of $s$ a constant value of about $75 \%$ is reached; in the Kubo-Greenwood description the magnetoresistance vanishes for $s>6$.

In order to illustrate this different behaviour, in figure 7, end-point-dependen t sheet resistances as defined in equations (30) and (32) are shown. From this figure, one can see that $\ln \left[\bar{r}_{(p)}\left(\mathrm{P} ; 2 n_{0}+s ; \delta\right)\right]$ increases linearly in the interior of the vacuum barrier with increasing number of vacuum layers, while because of the shape of the layer-resolved sheet resistance, having a maximum in the centre of the vacuum barrier (see figure 2), the quantity $\ln \left[r_{(p)}\left(\mathrm{P} ; 2 n_{0}+s ; \delta\right)\right]$ only shows a sharp increase in the first part of vacuum barrier. It should be noted that by choosing the end point $p$ in an arbitrary layer of the right lead, both $r_{(p)}(\mathrm{C} ; n)$ and $\bar{r}_{(p)}(\mathrm{C} ; n)$ remain almost unchanged, that is in both cases the sheet resistances are independent of the position of the contacts in the leads.

Abbreviating $r\left(\mathrm{C} ; 2 n_{0}+s ; \delta\right)$ simply by $r_{\mathrm{C}}(s)$ and assuming that the increase in $r_{\mathrm{C}}(s)$ with increasing $s$ is of an exponential form, that is

$$
r_{\mathrm{P}}(s)=A_{\mathrm{P}} \exp \left(K_{\mathrm{P}} s\right), \quad r_{\mathrm{AP}}(s)=A_{\mathrm{AP}} \exp \left(K_{\mathrm{AP}} s\right),
$$


(a)

(b)

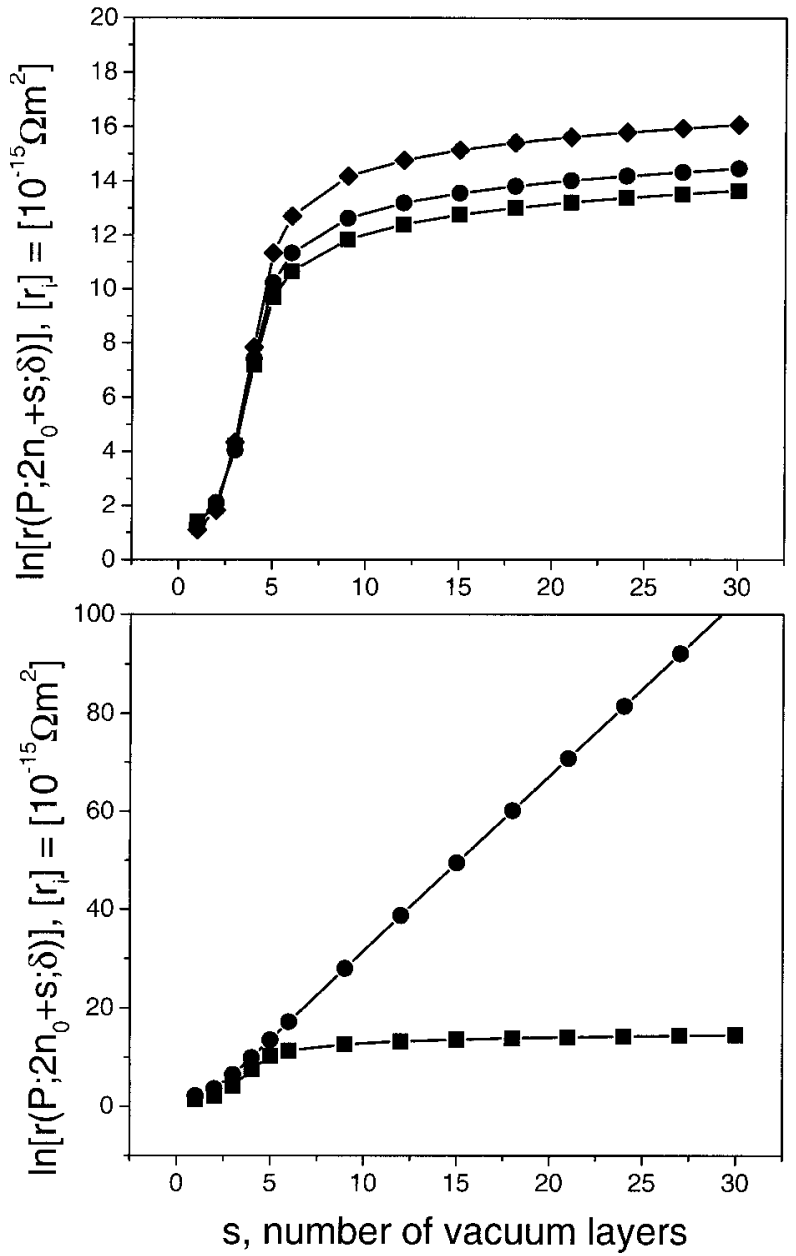

Figure 5. (a) $\ln \left[r\left(\mathrm{P} ; 2 n_{0}+s ; \delta\right)\right], n_{0} \geqslant 11$, as calculated in terms of the Kubo-Greenwood approach and displayed versus the number $s$ of vacuum layers: $(\diamond), \delta=0.1 \mathrm{mRy}$; $(\bullet), \delta=2.0 \mathrm{mRy}$; (ם), $\delta=3.0 \mathrm{mRy}$. (b) Comparison between the Landauer type (-) (equation (29)) and the Kubo-Greenwood type (苗) (equation (18)). Displayed is $\ln \left[r\left(\mathrm{P} ; 2 n_{0}+s ; \delta\right)\right], n_{0} \geqslant 11, \delta=2 \mathrm{mRy}$, as a function of the number $s$ of vacuum layers.

then quite clearly the corresponding magnetoresistance $R(s)$ is given by

$$
R(s)=1-C \exp \left[\left(K_{\mathrm{P}}-K_{\mathrm{AP}}\right) s\right],
$$

where $C=A_{\mathrm{P}} / A_{\mathrm{AP}}$. This now implies that the following cases have to be distinguished:

$$
\begin{aligned}
& K_{\mathrm{AP}}<K_{\mathrm{P}} \rightarrow \lim _{s \rightarrow \infty}[R(s)]=-\infty, \\
& K_{\mathrm{AP}}=K_{\mathrm{P}} \rightarrow \lim _{s \rightarrow \infty}[R(s)]=1-C \in[0,1), \\
& K_{\mathrm{AP}}>K_{\mathrm{P}} \rightarrow \lim _{s \rightarrow \infty}[R(s)]=1,
\end{aligned}
$$


(a)

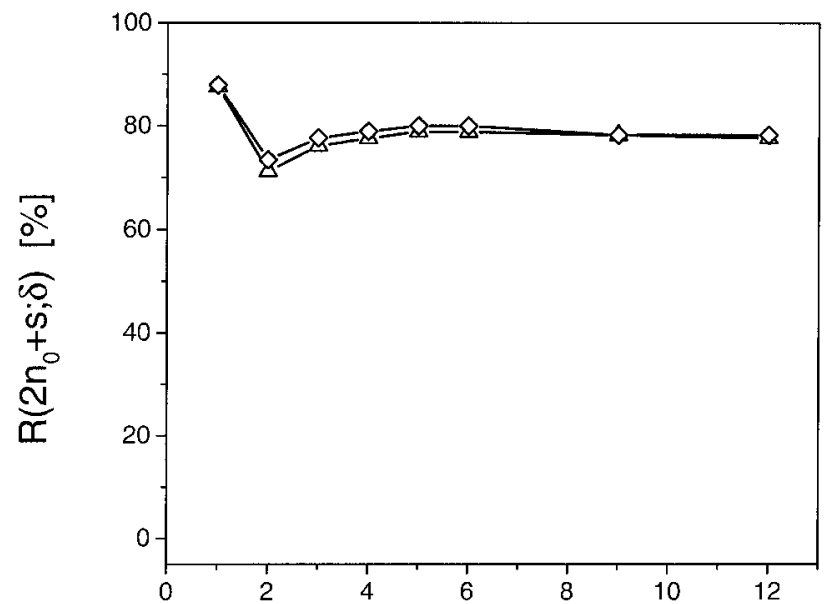

(b)

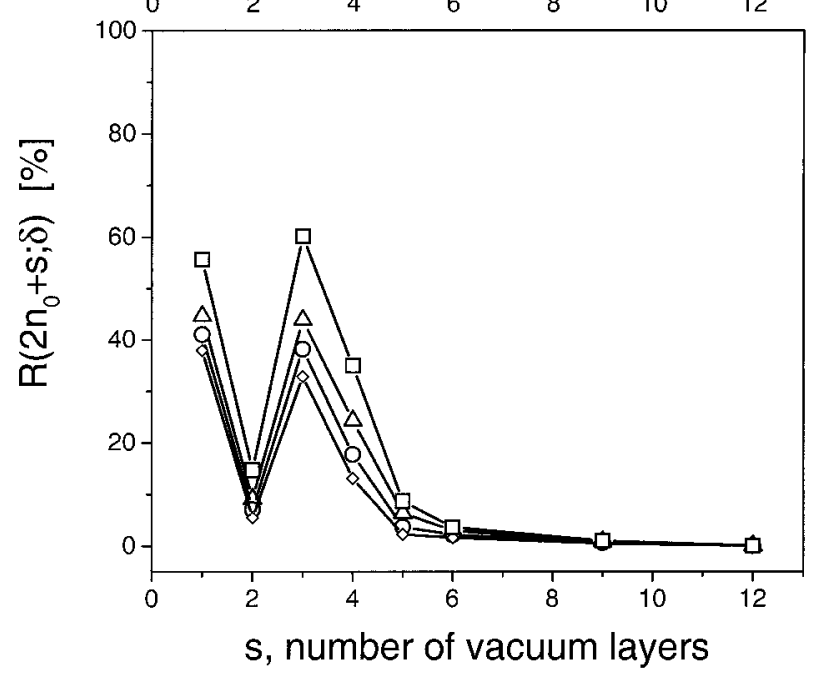

Figure 6. Magnetoresistance $R\left(2 n_{0}+s ; \delta\right), n_{0} \geqslant 11$, as described in terms of (a) equation (29) and $(b)$ equation (18) versus the number $s$ of vacuum layers: $(\mathrm{O}), \delta=0 \mathrm{mRy}$; $(\triangle), \delta=2 \mathrm{mRy} ;(\bigcirc), \delta=2.5 \mathrm{mRy} ;(\diamond), \delta=3 \mathrm{mRy}$.

leaving the conclusion that, with 'strict' exponential growth of the sheet resistance in the limit of infinitely separated magnetic leads, $R(s)$ tends to zero only in the particular case when $A_{\mathrm{P}}=A_{\mathrm{AP}}(C=1)$ and $K_{\mathrm{P}}=K_{\mathrm{AP}}$, that is $r_{\mathrm{P}}(s)=r_{\mathrm{AP}}(s)(\forall s)$.

As already seen in figure 2 from the layer-resolved sheet resistances, for $s=3$ a sudden increase in the magnetoresistance occurs; at $s=3$ the mode of electric transport seems to change dramatically; for $s>3$, however, the magnetoresistance drops again and vanishes beyond $s=9$. In order to explain this perhaps unexpected result, model calculations were performed for $s=12$ in which the (layer-resolved) Madelung potentials in the 'interior' of the vacuum barrier were modified by a constant:

$$
V_{\mathrm{M}}^{i}= \begin{cases}V_{\mathrm{M}}^{i}, & i \leqslant n_{0}+1, i \geqslant n_{0}+s-1, \\ V_{\mathrm{M}}^{i}+V_{\mathrm{C}}, & \text { otherwise. }\end{cases}
$$


(a)

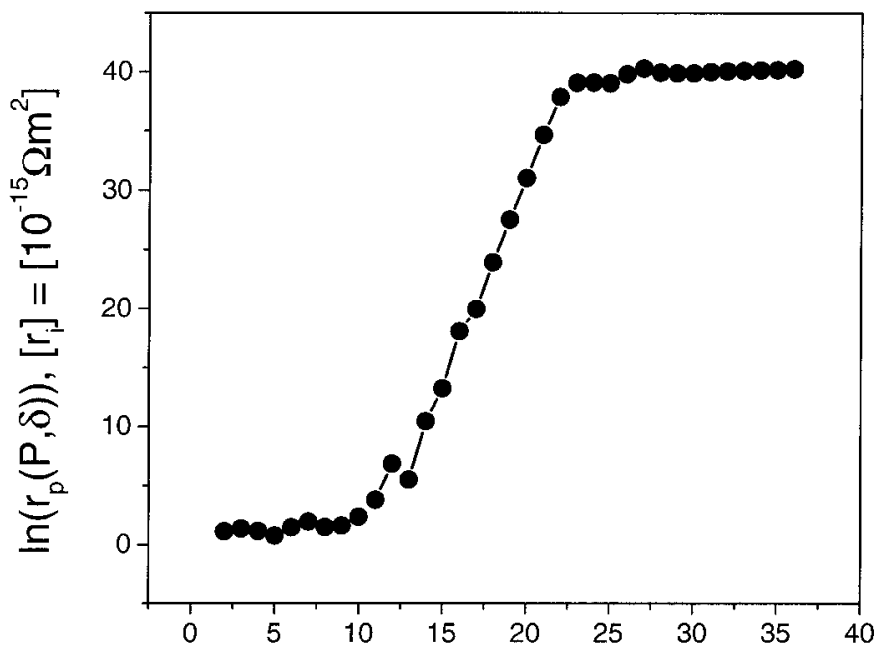

(b)

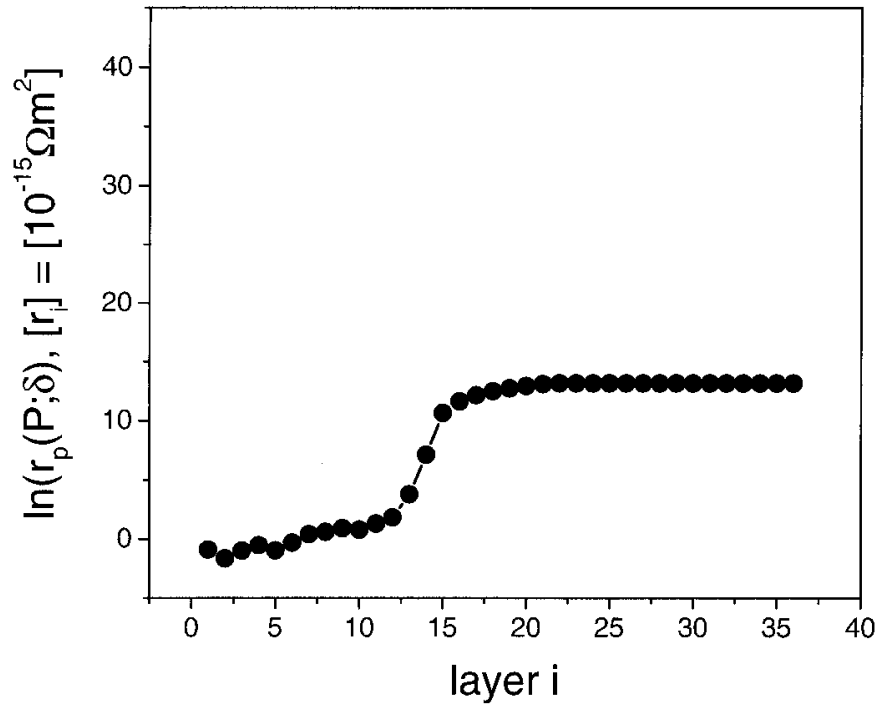

Figure 7. (a) End-point-dependent Landauer-type resistance (see equation (30)) and (b) the corresponding partial sums over layer-resolved sheet resistances (see equation (16)) for the parallel configuration of bcc $\mathrm{Fe}(100) / \mathrm{Fe}_{12} /$ vacuum $_{12} / \mathrm{Fe}_{12} / \mathrm{Fe}(100)$.

This procedure is illustrated in figure $8(a)$. Figure $8(b)$ shows that, for $V_{\mathrm{C}}>0$, $\ln \left[r\left(\mathbf{C} ; 2 n_{0}+s ; \delta\right)\right]$ first increases dramatically and then turns into a kind of asymptotic value for very high potential barriers. This asymptotic value is by orders of magnitude smaller than the value obtained from $\bar{r}\left(\mathrm{C} ; 2 n_{0}+s ; \delta\right)$ (see equation (29)). On the other hand for $V_{\mathrm{C}} \leqslant 0$ a cusp seems to exist. As can be seen from figure $9(a)$, by reducing the value of the potential barrier the layer-resolved sheet resistance immediately drops in the interior of the vacuum barrier and the magnetoresistance (bottom) increases. It should be noted that figure 9 offers an interesting aspect of the transition between metallic behaviour of electric transport and tunnelling; at $V_{\mathrm{M}}^{i}+V_{\mathrm{C}}=0$ there is a cusp that separates these two regimes of electric transport. Once $V_{\mathrm{M}}^{i}+V_{\mathrm{C}} \leqslant 0$ a situation as in a metal/metal heterojunction is reached. If 
(a)

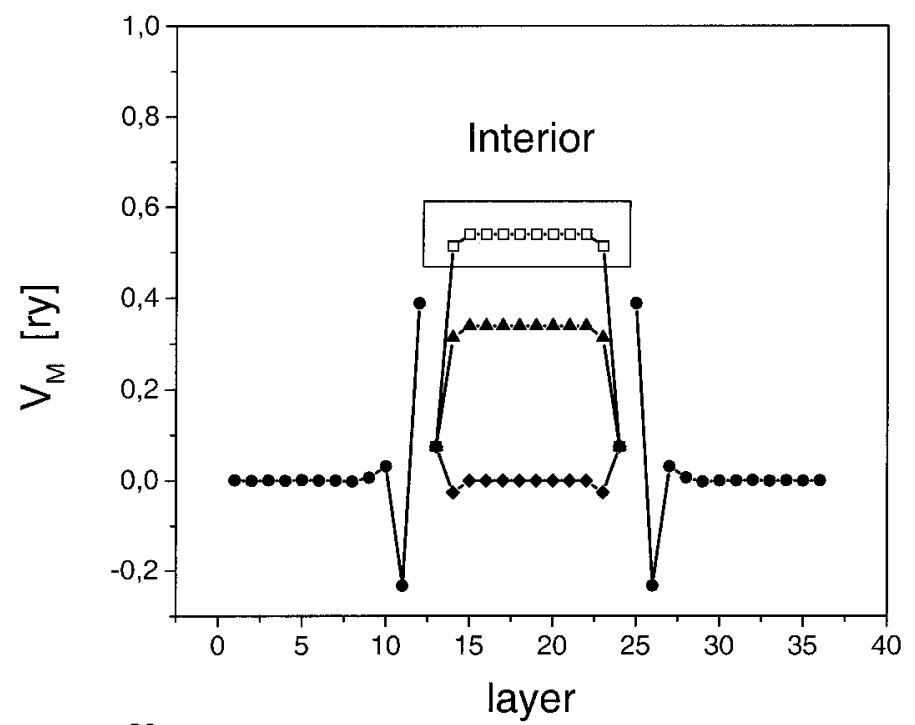

(b)

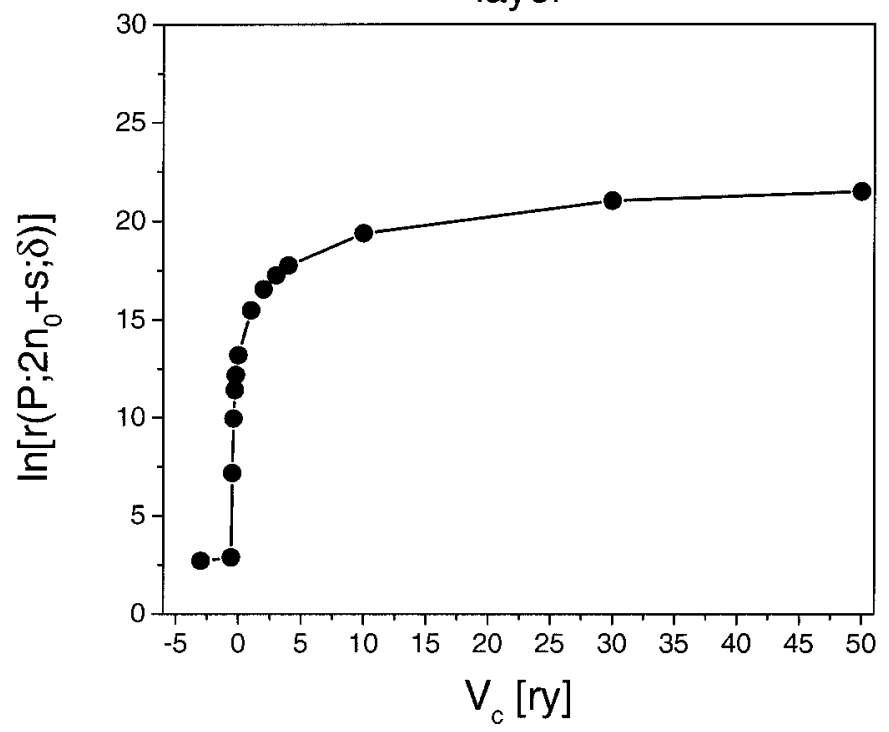

Figure 8. (a) Layer-resolved Madelung potentials and shifted potential barriers in the vacuum region of bcc $\mathrm{Fe}(100) / \mathrm{Fe}_{12} /$ vacuum $_{12} / \mathrm{Fe}_{12} / \mathrm{Fe}(100)$ (see also equation (41)): (๑), Fe; $(\square)$, vacuum region with $V_{\mathrm{C}}=0 \mathrm{Ry} ;(\boldsymbol{\Delta}), V_{\mathrm{C}}=-0.2 \mathrm{Ry} ;(\diamond)$, vacuum region with $V_{\mathrm{C}}=V_{\mathrm{M}}$. (b) $\ln \left[r\left(\mathrm{P} ; 2 n_{0}+s ; \delta\right)\right], n_{0} \geqslant 11, \delta=2 \mathrm{mRy}$, of bcc $\mathrm{Fe}(100) /$ $\mathrm{Fe}_{12} /$ vacuum $_{12} / \mathrm{Fe}_{12} / \mathrm{Fe}(100)$ as a function of $V_{\mathrm{C}}$.

$0 \leqslant V_{\mathrm{M}}^{i}+V_{\mathrm{C}} \leqslant V_{0}$, where $V_{0}$ is a reasonably small but positive constant, a tunnelling magnetoresistance can be expected even for rather very thick spacers, since the case of $s=12$ already corresponds to a spacer thickness of about $17 \AA$.

\subsection{The transmission matrix formalism}

As the results in $\S 7.1$ leave the question of the 'correct' asymptotic value of the magnetoresistance in the case of a vacuum barrier somewhat unanswered, also the transmission matrix formalism in terms of the TB LMTO method was applied, this 
(a)

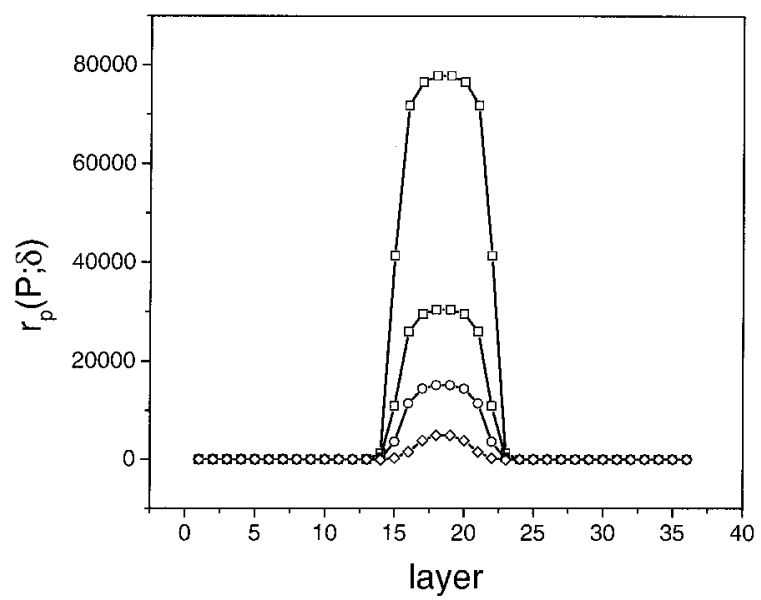

(b)

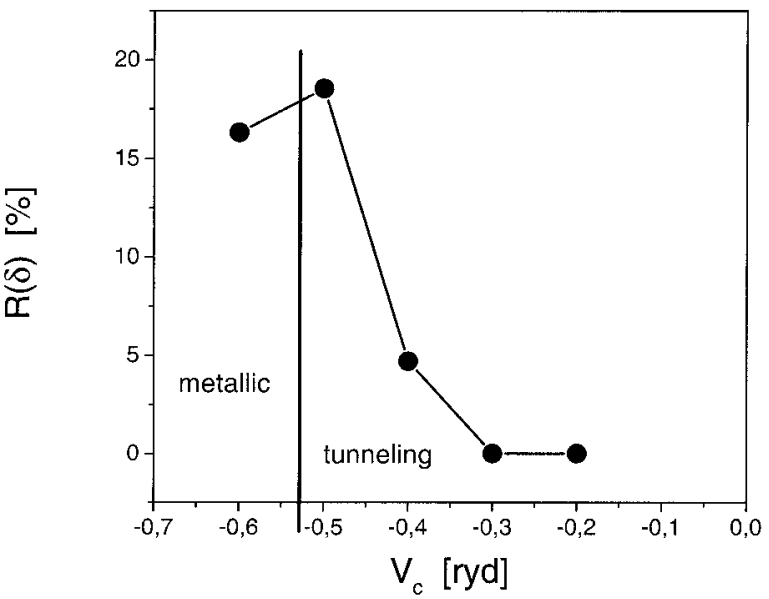

Figure 9. (a) Sheet resistance $r\left(\mathrm{P} ; 2 n_{0}+s ; \delta\right)\left((\square), V_{\mathrm{C}}=0 \mathrm{Ry} ;(\triangle), V_{\mathrm{C}}=0.2 \mathrm{Ry} ;(\bigcirc)\right.$, $\left.V_{\mathrm{C}}=0.3 \mathrm{Ry} ;(\diamond), V_{\mathrm{C}}=0.4 \mathrm{Ry}\right)$ and $(b)$ magnetoresistance $R\left(2 n_{0}+s ; \delta\right), \delta=2$ $\mathrm{mRy}$, in bcc $\mathrm{Fe}(100) / \mathrm{Fe}_{12} /$ vacuum $_{12} / \mathrm{Fe}_{12} / \mathrm{Fe}(100)$ as a function of the constant shift $V_{\mathrm{C}}$ (see also equation (41)). In $(b)$ the regimes of metallic and tunnelling behaviour of electric transport are separated by the condition $V_{\mathrm{M}}^{i}=-V_{\mathrm{C}}$ (vertical line).

time for a fcc $\mathrm{Co}(100) /$ vacuum $_{s} / \mathrm{Co}(100)$ heterojunction. In figure 10 the resistance (see also equation (21)) is shown versus the number of vacuum layers for the parallel as well as the antiparallel magnetic configuration, and in figure 11 the corresponding magnetoresistance. As can be easily seen from figure 11, the magnetoresistance has a minimum at $s=2$, has a maximum at $s=4$ and falls off for $s \geqslant 4$, indicating a vanishing magnetoresistance as $s$ becomes large. The shape of the magnetoresistance with respect to the number of vacuum layers is indeed very similar to the results obtained in terms of the Kubo-Greenwood approach for bcc $\mathrm{Fe}(001) / \operatorname{vacuum}_{s} /$ $\mathrm{Fe}(001)$ shown in figure 6 . This in turn implies that the 'visually obvious' exponential growth of the resistances in figure 10 is only approximate and that an asymptotic behaviour of the form

$$
\lim _{s \rightarrow \infty}\left[r_{\mathrm{AP}}(s)-r_{\mathrm{P}}(s)\right]=0
$$




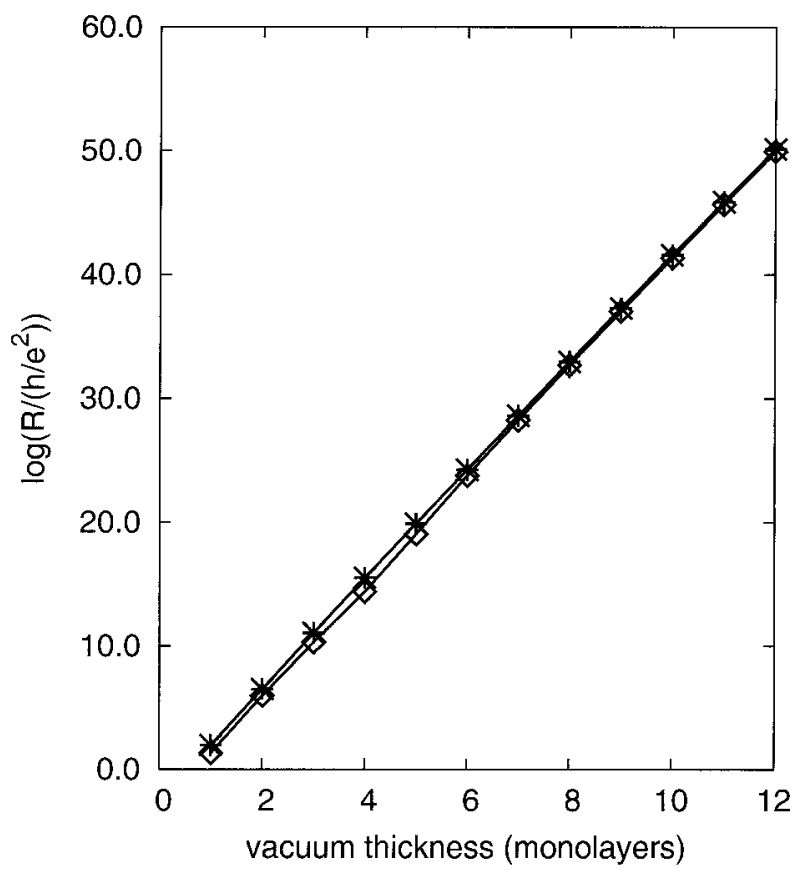

Figure 10. Parallel $(\square)$ and antiparallel (*) resistance of $\mathrm{Co}(100) /$ vacuum $_{s} / \mathrm{Co}(100)$ as calculated in terms of the transmission matrix formalism versus the number $s$ of vacuum layers.

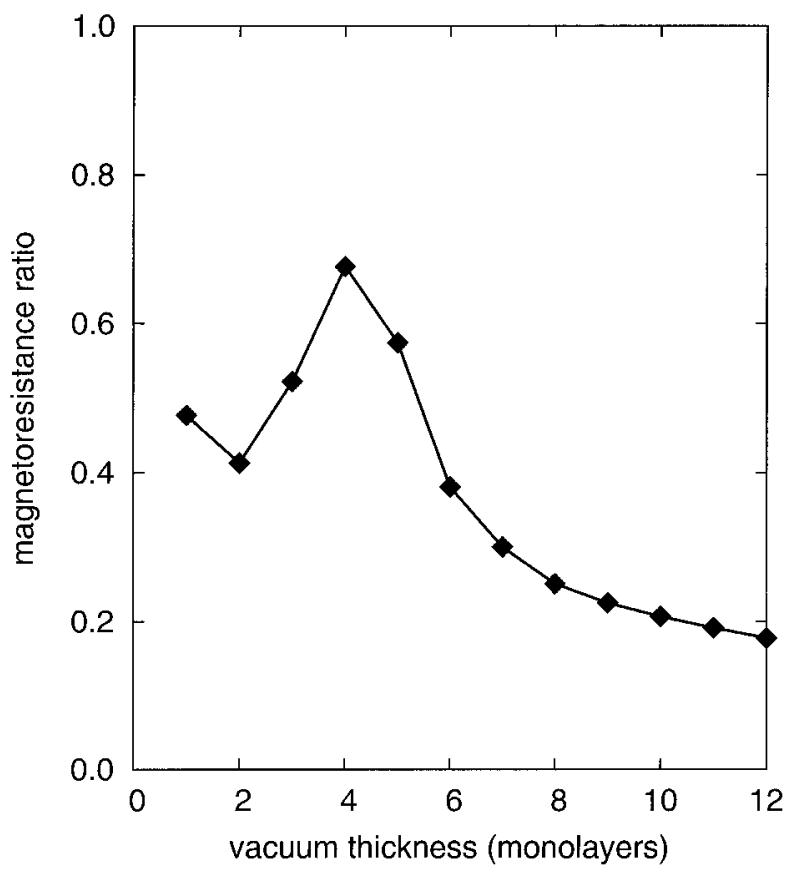

Figure 11. Magnetoresistance ratio of $\mathrm{Co}(100) /$ vacuum $_{s} / \mathrm{Co}(100)$ as calculated in terms of the transmission matrix formalism versus the number $s$ of vacuum layers. 
has to apply, which of course is the behaviour that one intuitively would expect as it appears unphysical to assume that two pieces of a magnetic metal separated by a 'truly macroscopic' distance would show a finite magnetoresistance. Since the TB LMTO calculations are not performed for self-consistent potentials, the similarity between figures 6 and 11 is the most important aspect of this comparison in here. The actual fall-off in the magnetoresistance with respect to $s$ depends slightly on the types of potential applied (self-consistency); also spin-orbit effects probably apply.

\section{$\S 8$. CONCLUSION}

It was shown in this paper that even the academic case of vacuum as an insulating spacer between Fe slabs offers interesting aspects of magnetotunnelling. First of all, as the thickness of the vacuum barrier increases, one reaches rather very quickly the case of free surfaces of the magnetic substrate that are stacked together but isolated. It should be noted that this is very reassuring since otherwise no proper description of free surfaces could be given. If, however, the potential barrier between the two slabs of magnetic material is sufficiently reduced, as can be the case in a realistic system, a reasonably large magnetoresistance is found. The size of the potential barrier therefore serves as one criterion for a tunnelling-type electric transport. The other criterion is of course the sign of the slope of the sheet resistances with respect to the imaginary part $\delta$ of the complex Fermi energy. Since $\delta$ can be viewed as a constant self-energy, a negative slope, that is decreasing sheet resistances with increasing $\delta$, is indicative of tunnelling, whereas the opposite applies for metalliclike electric transport. Furthermore, the concept of layer-resolved sheet resistances offers a clear visualization of the effects to be described. Most important, however, are the consequences for the magnetoresistance; both methods applied, namely the Kubo-Greenwood approach and the transmission matrix formulation, show that, when the vacuum barrier becomes very thick, the magnetoresistance tends to zero in qualitative agreement with the work of Mathon (1997).

Since, in this paper, two different kinds of ab-initio approach are compared, it is worthwhile to repeat certain aspects which make one or the other method more practicable in use for particular problems.

The use of the fully relativistic Kubo equation in terms of the screened KKR approach definitely has the disadvantage of being 'slow'. It assumes current conservation only for the total current. Furthermore, in the case of interdiffusion or alloying in the spacer the averaging of the off-diagonal scattering path operators is defined with respect to all layers and therefore the coherent potential approximation is reasonably good, in particular considering that the diagonal elements $\sigma_{\mu \mu}^{p p}\left(\mathrm{C} ; \varepsilon_{i}, \varepsilon_{j} ; n\right)$ are always by far the largest, the contribution from elements referring to larger values of $|p-q|$ being rather small (Blaas et al. 2002). In principle a calculation of vertex corrections is possible although at present numerically prohibitive. The same type of restriction applies to the use of a supercell approach; at present the numerical effort is insurmountable.

The transmission matrix formalism in terms of the TB LMTO method of course has the great advantage of being very fast not merely because only Green function matrix elements have to be calculated with respect to arbitrary chosen left and right lead layers. Since at present this approach is non-relativistic, current conservation for both 'spins' is required. As for thick spacers the relevant Green function matrix elements become very small; they have to be calculated with extreme care which, however, is only a matter of computational effort and therefore reduces to a 
technical problem. A disadvantage seems to be connected with the use of the coherent potential approximation when interdiffusion at interfaces is considered since, as was pointed out, the Green function matrix referring to the coherent potential medium does not give a full account of the scattering through the spacer when electrons travel from one (pure) lead to the other, that is vertex corrections have to be calculated. In this case the use of superlattices appears to be almost mandatory. This in turn of course saves evaluating the vertex corrections, but of course the use of supercells slows down the numerical efficiency and, for every system investigated, in principle the convergence with respect to the size (number of atoms per supercell) has to be checked.

\section{ACKNOWLEDGEMENTS}

This paper resulted from a collaboration partially funded by the RTN network 'Computational magnetoelectronics' (contract HPRN-CT-2000-00143) of the European Commission. Further financial support was provided by the Grant Agency of the Academy of Sciences of the Czech Republic (project A1010829), the Grant Agency of the Czech Republic (project 202/00/0122), the Ministry of Education, Youth, and Sports of the Czech Republic (COST P5.30), the Center for Computational Materials Science in Vienna (GZ 45.504) and the Országes Tudományos Kutatási Alapprogramok (contract OTKA T030240 and T029813).

\section{REFERENCES}

Akerman J., Schuller I. K., Slaughter, J. M., and Dave, R. W., 2001, Appl. Phys. Lett. 79, 3104.

Blaas, C., Szunyogh, L., Weinberger, P., Sommers, C., and Levy, P. M., 2001, Phys. Rev. B, 63, 224408.

Blats, C., Szunyogh, L., Weinberger, P., Sommers, C., Levy, P. M., and Shi, J., 2002, Phys. Rev. B (in the press).

Blats, C., Weinber ger, P., Szunyogh, L., Levy, P. M., and Sommers, C., 1999, Phys. Rev. B, 60, 492.

Butler, W. H., 1985, Phys. Rev. B, 31, 3260.

Greenwood, D. A., 1958, Proc. phys. Soc., 71, 585.

Kubo, R., 1957, J. phys. Soc. Japan, 12, 570.

Kubo, R., Myake, S. I., and Hashitsume, N., 1965, Solid St. Phys., 17, 288.

Kudrnovský, J., Drchal, V., BlaAs, C., Weinberger, P., Turek, I., and Bruno, P., 2000, Phys. Rev. B, 62, 15084.

Levy, P. M., 1994, Solid St. Phys., 47, 367.

Mathon, J., 1997, Phys. Rev. B, 56, 11810.

Nikolić, B. K., 2001, Phys. Rev. B, 64, 165303.

Nikolić, B. K., and Allen, P. B., 1999, Phys. Rev. B, 60, 3963; 2000, ibid., 60, 020201.

Rammer, J., 1998, Quantum Transport Theory (Reading, Massachusetts: Perseus).

Turek, I., Drchal, V., Kudrnovský, J., Sob, M., and Weinberger, P., 1997, Electronic Structure of Disordered Alloys, Surfaces and Interfaces (Boston, Massachusetts: Kluwer).

Turek, I., Kudrnovský, J., Drchal, V., Szunyogh, L., and Weinberger, P., 2002, Phys. Rev. B, (in the press).

Vosko, S. H., Wilk, L., and Nusair, M., 1980, Can. J. Phys., 58, 1200.

WeInberger, P., 1990, Electron Scattering Theory for Ordered and Disordered Matter (Oxford: Clarendon); 1997, Phil. Mag. B, 75, 509.

Weinberger, P., Levy, P. M., Banhart, J., Szunyogh, L., and Úfalussy, B., 1996, J. Phys.: condens. Matter, 8, 7677

Weinberger, P., and Szunyogh, L., 2000, Comput. Mater. Sci., 17, 414.

Weinberger, P., Szunyogh, L., Blahs, C., and Sommers, C., 2001, Phys. Rev. B, 64, 184429.

Weinberger, P., Turek, I., and Szunyogh, L., 1997, Int. J. quant. Chem., 63, 165.

Wunnicke, O., Papanikolaou, N., Zeller, R., Dededrichs, P. H., Drchal, V., and Kudr nOvsKÝ, J., 2001, Phys. Rev. Lett. (submitted). 